\title{
FIRST SEARCH FOR GRAVITATIONAL WAVES FROM THE YOUNGEST KNOWN NEUTRON STAR
}

J. Abadie ${ }^{1}$, B. P. Abbott ${ }^{1}$, R. Abbott ${ }^{1}$, M. Abernathy ${ }^{2}$, C. AdAms $^{3}$, R. Adhikari ${ }^{1}$, P. Ajith ${ }^{1}$, B. Allen ${ }^{4}, 5$, G. Allen $^{6}$,

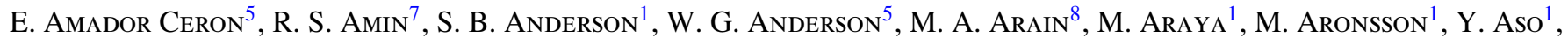
S. Aston ${ }^{9}$, D. E. AtKinson ${ }^{10}$, P. Aufmuth ${ }^{11}$, C. AulberT ${ }^{4}$, S. BabaK ${ }^{12}$, P. BAKer $^{13}$, S. Ballmer $^{1}$, D. Barker ${ }^{10}$, S. Barnum $^{14}$,

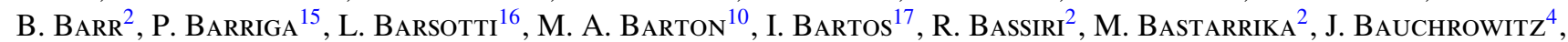

B. BehnKe ${ }^{12}$, M. Benacquista ${ }^{18}$, A. Bertolini ${ }^{4}$, J. Betzwieser ${ }^{1}$, N. Beveridge ${ }^{2}$, P. T. Beyersdorf ${ }^{19}$, I. A. BilenKo ${ }^{20}$,

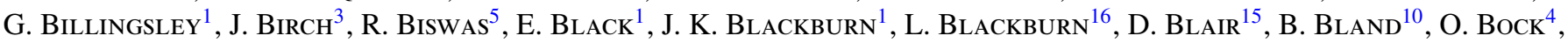

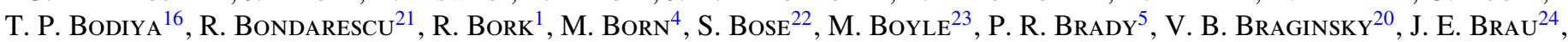
J. Breyer ${ }^{4}$, D. O. Bridges ${ }^{3}$, M. Brinkmann ${ }^{4}$, M. BritzGer ${ }^{4}$, A. F. Brooks ${ }^{1}$, D. A. Brown ${ }^{25}$, A. Buonanno ${ }^{26}$,

J. Burguet-Castell ${ }^{5}$, O. Burmeister ${ }^{4}$, R. L. Byer ${ }^{6}$, L. CAdonati ${ }^{27}$, J. B. CAMP ${ }^{28}$, P. CAMPSie ${ }^{2}$, J. CAnnizzo ${ }^{28}$,

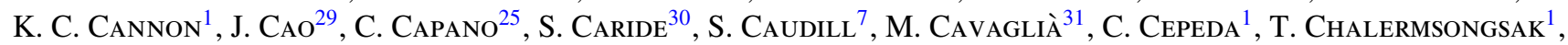
E. Chalkley ${ }^{2}$, P. Charlton ${ }^{32}$, S. Chelkowski ${ }^{9}$, Y. Chen $^{23}$, N. Christensen ${ }^{33}$, S. S. Y. Chua ${ }^{34}$, C. T. Y. Chung ${ }^{35}$, D. Clark ${ }^{6}$,

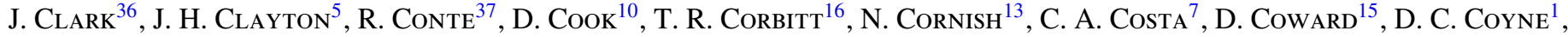

J. D. E. Creighton ${ }^{5}$, T. D. Creighton ${ }^{18}$, A. M. Cruise $^{9}$, R. M. Culter ${ }^{9}$, A. Cumming ${ }^{2}$, L. Cunningham ${ }^{2}$, K. Dahl $^{4}$, S. L. Danilishin ${ }^{20}$, R. Dannenberg ${ }^{1}$, K. Danzmann ${ }^{4}, 12$, K. Das $^{8}$, B. Daudert ${ }^{1}$, G. Davies ${ }^{36}$, A. Davis ${ }^{38}$, E. J. Daw ${ }^{39}$, T. Dayanga ${ }^{22}$, D. DeBra ${ }^{6}$, J. Degallaix ${ }^{4}$, V. Dergachev ${ }^{1}$, R. DeRosa ${ }^{7}$, R. DeSalvo $^{1}$, P. Devanka ${ }^{36}$, S. Dhurandhar $^{40}$, I. Di Palma ${ }^{4}$, M. Díaz ${ }^{18}$, F. Donovan ${ }^{16}$, K. L. Dooley ${ }^{8}$, E. E. Doomes ${ }^{41}$, S. Dorsher ${ }^{42}$, E. S. D. Douglas ${ }^{10}$, R. W. P. Drever ${ }^{43}$,

J. C. Driggers ${ }^{1}$, J. Dueck ${ }^{4}$, J.-C. Dumas ${ }^{15}$, T. Eberle ${ }^{4}$, M. Edgar $^{2}$, M. Edwards ${ }^{36}$, A. Effler ${ }^{7}$, P. Ehrens ${ }^{1}$, R. Engel ${ }^{1}$,

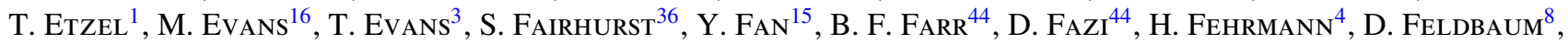
L. S. FinN ${ }^{21}$, M. Flanigan ${ }^{10}$, K. Flasch ${ }^{5}$, S. Foley ${ }^{16}$, C. Forrest ${ }^{45}$, E. Forsi ${ }^{3}$, N. Fotopoulos ${ }^{5}$, M. Frede ${ }^{4}$, M. Frei ${ }^{46}$, Z. Frei $^{47}$, A. Freise ${ }^{9}$, R. Frey ${ }^{24}$, T. T. Fricke ${ }^{7}$, D. Friedrich ${ }^{4}$, P. Fritschel ${ }^{16}$, V. V. Frolov ${ }^{3}$, P. Fulda ${ }^{9}$, M. FyfFe ${ }^{3}$, J. A. Garofoli ${ }^{25}$, I. Gholami ${ }^{12}$, S. Ghosh ${ }^{22}$, J. A. Giaime ${ }^{3,7}$, S. Giampanis ${ }^{4}$, K. D. Giardina ${ }^{3}$, C. Gill ${ }^{2}$, E. Goetz ${ }^{30}$,

L. M. GogGin ${ }^{5}$, G. GonZÁLEZ ${ }^{7}$, M. L. Gorodetsky ${ }^{20}$, S. Gossler $^{4}$, C. GraeF ${ }^{4}$, A. Grant ${ }^{2}$, S. Gras ${ }^{15}$, C. Gray ${ }^{10}$,

R. J. S. Greenhalgh ${ }^{48}$, A. M. Gretarsson ${ }^{38}$, R. Grosso ${ }^{18}$, H. Grote ${ }^{4}$, S. Grunewald ${ }^{12}$, E. K. Gustafson ${ }^{1}$, R. Gustafson ${ }^{30}$,

B. Hage ${ }^{11}$, P. Hall ${ }^{36}$, J. M. Hallam ${ }^{9}$, D. Hammer ${ }^{5}$, G. Hammond ${ }^{2}$, J. Hanks ${ }^{10}$, C. Hanna ${ }^{1}$, J. Hanson ${ }^{3}$, J. Harms ${ }^{42}$,

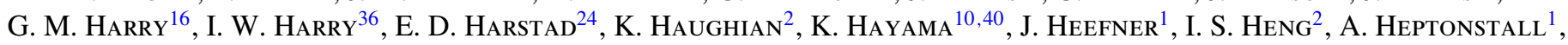

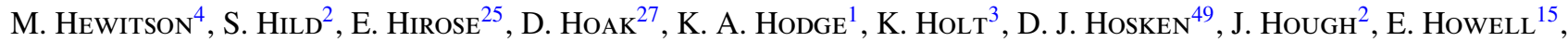

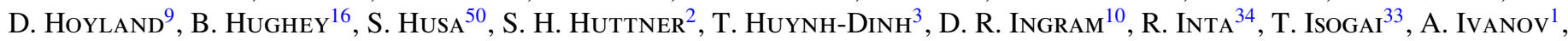

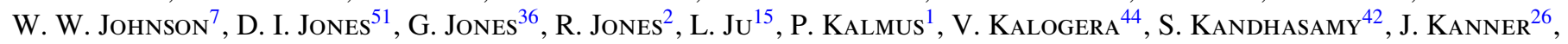

E. Katsavounidis ${ }^{16}$, K. Kawabe ${ }^{10}$, S. Kawamura ${ }^{52}$, F. Kawazoe ${ }^{4}$, W. Kells ${ }^{1}$, D. G. Keppel ${ }^{1}$, A. Khalaidovski ${ }^{4}$,

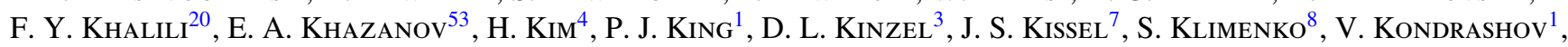
R. Kopparapu ${ }^{21}$, S. Koranda ${ }^{5}$, D. KozaK ${ }^{1}$, T. Krause ${ }^{46}$, V. Kringel ${ }^{4}$, S. Krishnamurthy ${ }^{44}$, B. Krishnan ${ }^{12}$, G. Kuehn ${ }^{4}$,

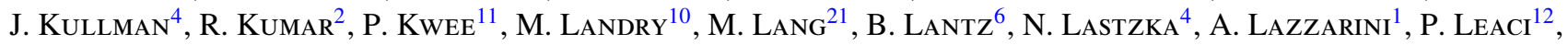

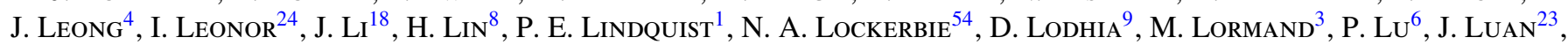

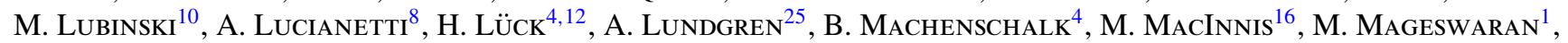
K. Mailand ${ }^{1}$, C. Mak ${ }^{1}$, I. Mandel ${ }^{44}$, V. Mandic ${ }^{42}$, S. Márka ${ }^{17}$, Z. Márka ${ }^{17}$, E. Maros ${ }^{1}$, I. W. Martin ${ }^{2}$, R. M. Martin ${ }^{8}$,

J. N. Marx ${ }^{1}$, K. Mason $^{16}$, F. Matichard ${ }^{16}$, L. Matone $^{17}$, R. A. Matzner ${ }^{46}$, N. Mavalvala ${ }^{16}$, R. McCarthy $^{10}$,

D. E. McClelland ${ }^{34}$, S. C. McGuire ${ }^{41}$, G. McIntyre ${ }^{1}$, G. McIvor ${ }^{46}$, D. J. A. McKechan ${ }^{36}$, G. Meadors ${ }^{30}$, M. Mehmet ${ }^{4}$, T. Meier ${ }^{11}$, A. Melatos ${ }^{35}$, A. C. Melissinos ${ }^{45}$, G. Mendell ${ }^{10}$, D. F. Menéndez ${ }^{21}$, R. A. Mercer ${ }^{5}$, L. Merill ${ }^{15}$, S. Meshioov ${ }^{1}$, C. Messenger ${ }^{4}$, M. S. Meyer ${ }^{3}$, H. Miao ${ }^{15}$, J. Miller ${ }^{2}$, Y. Mino ${ }^{23}$, S. Mitra ${ }^{1}$, V. P. Mitrofanov ${ }^{20}$, G. Mitselmakher ${ }^{8}$,

R. Mittleman ${ }^{16}$, B. Moe $^{5}$, S. D. Mohanty ${ }^{18}$, S. R. P. Mohapatra ${ }^{27}$, D. Morard ${ }^{10}$, G. Moreno ${ }^{10}$, T. Morioka ${ }^{52}$, K. Mors',

K. Mossavi ${ }^{4}$, C. Mowlowry ${ }^{34}$, G. Mueller ${ }^{8}$, S. Mukherjee ${ }^{18}$, A. Mullavey ${ }^{34}$, H. Müller-Ebhardt ${ }^{4}$, J. Munch $^{49}$,

P. G. MurraY ${ }^{2}$, T. Nash ${ }^{1}$, R. Nawrodt ${ }^{2}$, J. Nelson ${ }^{2}$, G. Newton ${ }^{2}$, A. Nishizawa ${ }^{52}$, D. Nolting ${ }^{3}$, E. Ochsner ${ }^{26}$, J. O’ Dell $^{48}$,

G. H. Ogin ${ }^{1}$, R. G. OldenburG ${ }^{5}$, B. O’Reilly ${ }^{3}$, R. O’'Shaughnessy ${ }^{21}$, C. Osthelder ${ }^{1}$, D. J. OtTaway ${ }^{49}$, R. S. Ottens ${ }^{8}$,

H. Overmier ${ }^{3}$, B. J. Owen ${ }^{21}$, A. Page ${ }^{9}$, Y. Pan ${ }^{26}$, C. Pankow ${ }^{8}$, M. A. Papa ${ }^{5,13}$, M. Pareja ${ }^{4}$, P. Patel ${ }^{1}$, M. Pedraza ${ }^{1}$, L. Pekowsky ${ }^{25}$, S. Penn ${ }^{55}$, C. Peralta ${ }^{12}$, A. Perreca ${ }^{9}$, M. PickenPack ${ }^{4}$, I. M. Pinto ${ }^{56}$, M. Pitkin ${ }^{2}$, H. J. Pletsch ${ }^{4}$, M. V. Plissi ${ }^{2}$, F. Postiglione ${ }^{37}$, V. Predo ${ }^{36}$, L. R. Price ${ }^{5}$, M. Prijatelj ${ }^{4}$, M. Principe ${ }^{56}$, R. Prix $^{4}$, L. Prokhorov ${ }^{20}$, O. Puncken ${ }^{4}$, V. QuetschKe ${ }^{18}$, F. J. RaAB ${ }^{10}$, T. RAdKe ${ }^{12}$, H. RadKins ${ }^{10}$, P. RafFaI ${ }^{47}$, M. RaKhmanov ${ }^{18}$, B. Rankins ${ }^{31}$,

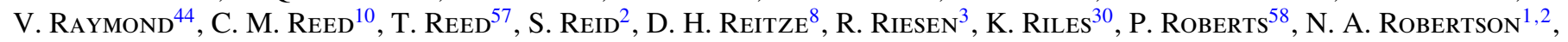
C. Robinson ${ }^{36}$, E. L. Robinson ${ }^{12}$, S. RodDY ${ }^{3}$, C. Röver ${ }^{4}$, J. Rollins ${ }^{17}$, J. D. Romano ${ }^{18}$, J. H. Romie ${ }^{3}$, S. Rowan ${ }^{2}$, A. RÜDiger ${ }^{4}$, K. Ryan ${ }^{10}$, S. Sakata ${ }^{52}$, M. Sakosky ${ }^{10}$, F. SAlemi ${ }^{4}$, L. SAmmuT ${ }^{35}$, L. Sancho de La Jordana ${ }^{50}$, V. Sandberg ${ }^{10}$,

V. Sannibale ${ }^{1}$, L. Santamaría ${ }^{12}$, G. Santostasi ${ }^{59}$, S. Saraf ${ }^{14}$, B. S. SathyapraKash ${ }^{36}$, S. Sato $^{52}$, M. Satterthwaite ${ }^{34}$,

P. R. Saulson ${ }^{25}$, R. SAVAGE ${ }^{10}$, R. Schilling ${ }^{4}$, R. Schnabel ${ }^{4}$, R. Schofield ${ }^{24}$, B. Schulz ${ }^{4}$, B. F. Schutz ${ }^{13,37}$,

P. Schwinkerg ${ }^{10}$, J. ScotT ${ }^{2}$, S. M. ScotT ${ }^{34}$, A. C. Searle ${ }^{1}$, F. Seifert ${ }^{1}$, D. Sellers ${ }^{3}$, A. S. Sengupta ${ }^{1}$, A. Sergeev ${ }^{53}$,

D. Shaddock ${ }^{34}$, B. Shapiro ${ }^{16}$, P. Shawhan ${ }^{26}$, D. H. ShoemaKer ${ }^{16}$, A. Sibley ${ }^{3}$, X. Siemens ${ }^{5}$, D. SigG ${ }^{10}$, A. Singer ${ }^{1}$,

A. M. Sintes ${ }^{50}$, G. Skelton ${ }^{5}$, B. J. J. Slagmolen ${ }^{34}$, J. Slutsky ${ }^{7}$, J. R. Smith ${ }^{60}$, M. R. Smith ${ }^{1}$, N. D. Smith ${ }^{16}$, K. SomiYa ${ }^{23}$, 
B. SoraZu ${ }^{2}$, F. C. Speirits ${ }^{2}$, A. J. Stein ${ }^{16}$, L. C. Stein ${ }^{16}$, S. Steinlechner ${ }^{4}$, S. StePlewski ${ }^{22}$, A. Stochino ${ }^{1}$, R. Stone ${ }^{18}$, K. A. Strain ${ }^{2}$, S. Strigin ${ }^{20}$, A. Stroeer ${ }^{28}$, A. L. Stuver ${ }^{3}$, T. Z. Summerscales ${ }^{58}$, M. Sung ${ }^{7}$, S. Susmithan ${ }^{15}$, P. J. Sutton ${ }^{36}$,

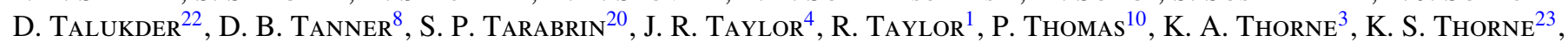

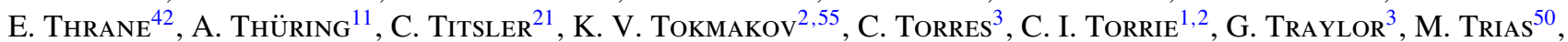

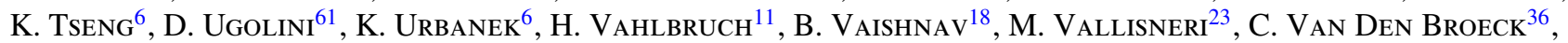
M. V. van der Sluys ${ }^{44}$, A. A. van VegGel ${ }^{2}$, S. Vass ${ }^{1}$, R. Vaulin ${ }^{5}$, A. Vecchio ${ }^{9}$, J. Veitch ${ }^{36}$, P. J. Veitch ${ }^{49}$, C. VeltKamp ${ }^{4}$, A. Villar ${ }^{1}$, C. Vorvick ${ }^{10}$, S. P. Vyachanin ${ }^{20}$, S. J. Waldman ${ }^{16}$, L. Wallace ${ }^{1}$, A. Wanner ${ }^{4}$, R. L. Ward ${ }^{1}$, P. Wei $^{25}$, M. Weinert ${ }^{4}$, A. J. Weinstein ${ }^{1}$, R. Weiss ${ }^{16}$, L. Wen ${ }^{16,62}$, S. Wen ${ }^{7}$, P. Wessels ${ }^{4}$, M. West ${ }^{25}$, T. Westphal ${ }^{4}$, K. Wette $^{34}$, J. T. Whelan ${ }^{62}$, S. E. Whitcomb ${ }^{1}$, D. J. White ${ }^{39}$, B. F. Whiting ${ }^{8}$, C. Wilkinson ${ }^{10}$, P. A. Willems ${ }^{1}$, L. Williams ${ }^{8}$,

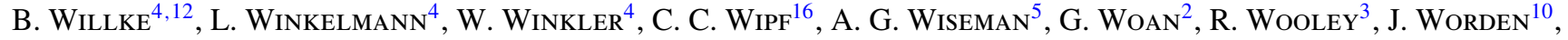
I. Yakushin ${ }^{3}$, H. Yamamoto ${ }^{1}$, K. Yamamoto ${ }^{4}$, D. Yeaton-Massey ${ }^{1}$, S. Yoshida ${ }^{63}$, P. P. Yu ${ }^{5}$, M. Zanolin ${ }^{38}$, L. Zhang ${ }^{1}$, Z. ZhaNG ${ }^{15}$, C. ZhaO ${ }^{15}$, N. Zotov ${ }^{57}$, M. E. ZuCKeR ${ }^{16}$, AND J. ZweIZIG ${ }^{1}$

(The LiGO ScIENTIFIC COLlaboration ${ }^{64}$ )

${ }^{1}$ LIGO-California Institute of Technology, Pasadena, CA 91125, USA

${ }^{2}$ University of Glasgow, Glasgow, G12 8QQ, UK

${ }^{3}$ LIGO-Livingston Observatory, Livingston, LA 70754, USA

${ }^{4}$ Albert-Einstein-Institut, Max-Planck-Institut für Gravitationsphysik, D-30167 Hannover, Germany

${ }^{5}$ University of Wisconsin-Milwaukee, Milwaukee, WI 53201, USA ${ }^{6}$ Stanford University, Stanford, CA 94305, USA

${ }^{7}$ Louisiana State University, Baton Rouge, LA 70803, USA

${ }^{8}$ University of Florida, Gainesville, FL 32611, USA

${ }^{9}$ University of Birmingham, Birmingham, B15 2TT, UK

${ }^{10}$ LIGO-Hanford Observatory, Richland, WA 99352, USA

${ }^{11}$ Leibniz Universität Hannover, D-30167 Hannover, Germany

${ }^{12}$ Albert-Einstein-Institut, Max-Planck-Institut für Gravitationsphysik, D-14476 Golm, Germany

${ }_{13}$ Montana State University, Bozeman, MT 59717, USA

${ }^{14}$ Sonoma State University, Rohnert Park, CA 94928, USA

${ }^{15}$ University of Western Australia, Crawley WA 6009, Australia

${ }^{16}$ LIGO-Massachusetts Institute of Technology, Cambridge, MA 02139, USA

${ }^{17}$ Columbia University, New York, NY 10027, USA

18 The University of Texas at Brownsville and Texas Southmost College, Brownsville, TX 78520, USA

${ }^{19}$ San Jose State University, San Jose, CA 95192, USA

${ }^{20}$ Moscow State University, Moscow, 119992, Russia

${ }^{21}$ The Pennsylvania State University, University Park, PA 16802, USA

${ }^{22}$ Washington State University, Pullman, WA 99164, USA

${ }^{23}$ Caltech-CaRT, Pasadena, CA 91125, USA

${ }^{24}$ University of Oregon, Eugene, OR 97403, USA

${ }^{25}$ Syracuse University, Syracuse, NY 13244, USA

${ }^{26}$ University of Maryland, College Park, MD 20742, USA

${ }^{27}$ University of Massachusetts-Amherst, Amherst, MA 01003, USA

${ }^{28}$ NASA/Goddard Space Flight Center, Greenbelt, MD 20771, USA

${ }^{29}$ Tsinghua University, Beijing 100084 , China

${ }^{30}$ University of Michigan, Ann Arbor, MI 48109, USA

31 The University of Mississippi, University, MS 38677, USA

${ }^{32}$ Charles Sturt University, Wagga Wagga, NSW 2678, Australia

${ }^{33}$ Carleton College, Northfield, MN 55057, USA

${ }^{34}$ Australian National University, Canberra, 0200, Australia

35 The University of Melbourne, Parkville VIC 3010, Australia ${ }^{36}$ Cardiff University, Cardiff, CF24 3AA, UK

${ }^{37}$ University of Salerno, 84084 Fisciano (Salerno), Italy

${ }^{38}$ Embry-Riddle Aeronautical University, Prescott, AZ 86301, USA

${ }^{39}$ The University of Sheffield, Sheffield S10 2TN, UK

${ }^{40}$ Inter-University Centre for Astronomy and Astrophysics, Pune 411 007, India

${ }^{41}$ Southern University and A\&M College, Baton Rouge, LA 70813, USA

${ }^{42}$ University of Minnesota, Minneapolis, MN 55455, USA

${ }^{43}$ California Institute of Technology, Pasadena, CA 91125, USA

${ }^{44}$ Northwestern University, Evanston, IL 60208, USA

${ }^{45}$ University of Rochester, Rochester, NY 14627, USA

46 The University of Texas at Austin, Austin, TX 78712, USA

${ }^{47}$ Eötvös University, ELTE 27 Budapest, Hungary

${ }^{48}$ Rutherford Appleton Laboratory, HSIC, Chilton, Didcot, Oxon OX11 0QX, UK

${ }^{49}$ University of Adelaide, Adelaide, SA 5005, Australia

${ }^{50}$ Universitat de les Illes Balears, E-07122 Palma de Mallorca, Spain

${ }^{51}$ University of Southampton, Southampton, SO17 1BJ, UK

${ }^{52}$ National Astronomical Observatory of Japan, Tokyo 181-8588, Japan

${ }^{53}$ Institute of Applied Physics, Nizhny Novgorod, 603950, Russia

${ }^{54}$ University of Strathclyde, Glasgow, G1 1XQ, UK

${ }^{55}$ Hobart and William Smith Colleges, Geneva, NY 14456, USA

${ }^{56}$ University of Sannio at Benevento, I-82100 Benevento, Italy

${ }^{57}$ Louisiana Tech University, Ruston, LA 71272, USA 


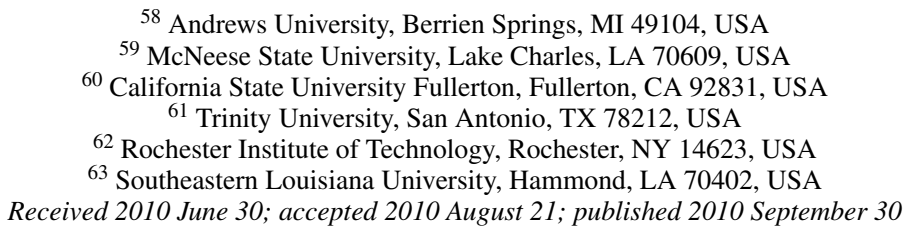

\begin{abstract}
We present a search for periodic gravitational waves from the neutron star in the supernova remnant Cassiopeia A. The search coherently analyzes data in a 12 day interval taken from the fifth science run of the Laser Interferometer Gravitational-Wave Observatory. It searches gravitational-wave frequencies from $100 \mathrm{Ho} 300 \mathrm{~Hz}$ and covers a wide range of first and second frequency derivatives appropriate for the age of the remnant and for different spin-down mechanisms. No gravitational-wave signal was detected. Within the range of search frequencies, we set $95 \%$ confidence upper limits of $(0.7-1.2) \times 10^{-24}$ on the intrinsic gravitational-wave strain, (0.4-4) $\times 10^{-4}$ on the equatorial ellipticity of the neutron star, and $0.005-0.14$ on the amplitude of $r$-mode oscillations of the neutron star. These direct upper limits beat indirect limits derived from energy conservation and enter the range of theoretical predictions involving crystalline exotic matter or runaway $r$ modes. This paper is also the first gravitational-wave search to present upper limits on the $r$-mode amplitude.
\end{abstract}

Key words: gravitational waves - stars: neutron - supernovae: individual (Cassiopeia A)

\section{INTRODUCTION}

Using data from the Laser Interferometer Gravitational-Wave Observatory (LIGO; Abbott et al. 2009d), the LIGO Scientific Collaboration, and Virgo Collaboration have published searches for periodic gravitational waves from three astrophysically distinct types of rapidly rotating neutron star. Searches have targeted non-accreting pulsars (Abbott et al. 2004b, 2005b, 2007c, 2010), most notably the Crab pulsar (Abbott et al. 2008 b, 2010), using data from LIGO's first five science runs (designated S1-S5). Two searches have targeted the accreting neutron star in the low-mass X-ray binary Scorpius X-1, using data from S2 (Abbott et al. 2007a) and S4 (Abbott et al. 2007b). Other searches have been broadband all-sky surveys for as-yet undiscovered neutron stars, using data from S2 (Abbott et al. 2005a, 2007a), S4 (Abbott et al. 2008a, 2009a), and S5 (Abbott et al. 2009b, 2009c).

In this paper, we present the first directed search for periodic gravitational waves from a known, isolated, non-pulsing neutron star. The search targets the central compact object (CCO) in the supernova remnant Cassiopeia A (Cas A). The remnant is estimated to be $3.4_{-0.1}^{+0.3} \mathrm{kpc}$ distant (Reed et al. 1995) and to have been born in the year $1681 \pm 19$ (Fesen et al. 2006b). It is the second-youngest known supernova remnant in the Galaxy, and the youngest with a confirmed CCO (Reynolds et al. 2008; De Luca 2008). The remnant and CCO have been extensively studied through electromagnetic observations, which we summarize in Section 1.1.

There is compelling evidence that the Cas A CCO is a neutron star (Pavlov et al. 2000; Chakrabarty et al. 2001; Gotthelf \& Halpern 2008; Pavlov \& Luna 2009; Ho \& Heinke 2009). We argue, in Section 1.2, that its age and youth make it an interesting target for a search for periodic gravitational waves. Its youth means that it has not been covered by all-sky surveys for periodic gravitational waves, which focus on spin-down timescales much longer than the age of the remnant (Abbott et al. 2005a, 2007a, 2008a, 2009a, 2009b, 2009c). We describe the first search for gravitational waves from Cas A in Section 2, present the results of the search in Section 3, and discuss them in Section 4. (We

\footnotetext{
${ }^{64}$ http://www.ligo.org
}

shall often abbreviate "the Cas A central compact object" to "Cas A.")

Since the rotation frequency, and hence the gravitationalwave frequency, of Cas A is unknown, we search for periodic gravitational waves with frequencies between 100 and $300 \mathrm{~Hz}$. At these frequencies, where the strain noise of the LIGO detectors is lowest, the search is designed to beat indirect upper limits on gravitational radiation based on energy conservation (see Wette et al. 2008, and Sections 1.2 and 2.3). The search found no credible signal (see Section 3). In the absence of a detection, we present $95 \%$ confidence upper limits on a gravitational-wave signal from Cas A, assuming its frequency is within the searched band. Upper limits are given for the intrinsic gravitational-wave strain $h_{0}$, the equatorial ellipticity $\epsilon$, and the $r$-mode amplitude $\alpha$.

Within the searched frequency band, the upper limits presented in this paper beat the indirect upper limits, as expected. Cas A is now one of only a handful of neutron stars (see Abbott et al. 2010) where the most sensitive upper limits on gravitational radiation have been obtained using gravitational-wave detectors such as LIGO. This paper is also the first gravitationalwave search to present upper limits on the $r$-mode amplitude. The best upper limits on $\epsilon$ (a few times $10^{-5}$ ) and $\alpha$ (a few times $10^{-3}$ ) are within the range of some theoretical predictions (see Section 4).

\subsection{Electromagnetic Observations}

The Cas A CCO was first discovered as an X-ray point source in first-light images taken by the Chandra X-ray Observatory (Tananbaum 1999). It was subsequently identified in other satellite data dating back to the year 1979; the X-ray flux appears to have been constant since then (Pavlov et al. 2000). Optical and near-infrared searches have not found the CCO, and have all but ruled out the presence of an accretion disk from fallback, and a binary companion (Ryan et al. 2001; Kaplan et al. 2001; Fesen et al. 2006a; Wang et al. 2007). The absence of the latter is puzzling, since the light-echo spectrum of the supernova, of type IIb, implies that the progenitor was stripped of hydrogen by a companion (Young et al. 2006; Krause et al. 2008). One possibility is that the progenitor was the product of 
binary companions merging during a common envelope phase (Krause et al. 2008).

The CCO appears to be a neutron star with a low surface magnetic field (an anti-magnetar). Blackbody fits to the X-ray spectrum by Pavlov et al. (2000) and Chakrabarty et al. (2001) implied a temperature too high, and an emitting area too small, to be consistent with emission from the whole surface of a cooling neutron star, or from the inner region of an accretion disk around a black hole. Pavlov et al. (2000) proposed a model of a strongly magnetized neutron star (a magnetar) with hot polar caps, which would, however, lead to X-ray pulsations which have not been observed (see below). Light echoes from the explosion have been interpreted as signs of a flare, reminiscent of a soft gamma repeater, occurring in the year 1953 (Krause et al. 2005). Such a flare, if single, could also have been due to a one-time phase transition (Gotthelf \& Halpern 2008). More recently, however, this interpretation of the echoes, and thus the evidence for the flare, has been discounted (Kim et al. 2008; Dwek \& Arendt 2008). Recently, Ho \& Heinke (2009) combined previous X-ray spectra (Hwang et al. 2004; Pavlov \& Luna 2009), carefully adjusted for instrumental effects, and fitted them to various light-element atmosphere models. Their best fit was for nearly isotropic emission from the entire surface of a neutron star with a carbon atmosphere, low magnetic field, and mass and radius within normal ranges.

The spin period of the neutron star is unknown. McLaughlin et al. (2001) searched for radio pulses (including possible binary orbital periods as short as a few hours) and found none at periods as short as $1-10 \mathrm{~ms}$, depending on dispersion measure, making the $\mathrm{CCO}$ much more radio quiet than any known radio pulsar under $10^{4}$ years old. Searches for X-ray pulsations at periods as short as $2 \mathrm{~ms}$ (Chakrabarty et al. 2001; Murray et al. 2002; Mereghetti et al. 2002; Pavlov \& Luna 2009) have produced at best a marginal candidate for a period at $12 \mathrm{~ms}$, which has not been confirmed. No pulsar wind nebula has been detected (Hwang et al. 2004; Pavlov \& Luna 2009).

\subsection{Motivation for a Gravitational-wave Search}

If the $\mathrm{CCO}$ in Cas A is an anti-magnetar, it may be spinning fast enough to emit periodic gravitational waves above $100 \mathrm{~Hz}$, where LIGO is most sensitive.

To date, seven supernova remnant CCOs are known: three have observed spin periods and a fourth has an observed periodicity that may be due to binary orbital motion (De Luca 2008; Gotthelf \& Halpern 2009). The fastest-spinning CCOs have rotation periods of $\sim 100 \mathrm{~ms}$, which are much slower than the longest rotation periods covered by this search $(20 \mathrm{~ms}$ for gravitational waves from a non-axisymmetric distortion and $13 \mathrm{~ms}$ for $r$-modes; see Section 3). Only one of the CCOs has a measurable spin-down (Halpern \& Gotthelf 2010) and the others have tight upper limits. This indicates that the CCOs have magnetic fields much less than those of typical radio pulsars, and rotation periods that have not changed significantly since birth. If Cas A spins as slowly and constantly as these objects, it is not detectable by LIGO. It is difficult, however, to definitively extrapolate the general properties of CCOs from a sample of three or four.

Young neutron stars, such as Cas A, may be the most likely to retain non-axisymmetries from the violent circumstances of their births. For example, the formation of the crust during an epoch of perturbations such as $r$-modes (Lindblom et al. 2000; Wu et al. 2001) could lead to an irregular shape. Gravitational radiation may also be generated by the continuing non- axisymmetry of the $r$-modes themselves (Owen et al. 1998), which may last for up to thousands of years (Arras et al. 2003) depending on the composition and viscosity of the star.

Indirect upper limits on gravitational-wave emission from Cas A can be estimated using a method similar to the spin-down limit for known pulsars (Wette et al. 2008; Owen 2010). If the star was born spinning at least $\sim 20 \%$ more rapidly than it is now, and the spin-down evolution has been dominated by the emission of gravitational waves, the unknown spin frequency and frequency derivative can be eliminated in favor of the known age to place a rough upper limit on the gravitationalwave emission. For Cas A, the indirect limit on the intrinsic gravitational-wave strain $h_{0}$ (see Section 2.2) is

$$
h_{0} \lesssim 1.2 \times 10^{-24} D_{3.4}^{-1} \tau_{300}^{-1 / 2} I_{45}^{1 / 2},
$$

where $D_{3.4}$ is the distance to Cas A in units of $3.4 \mathrm{kpc}, \tau_{300}$ is its age in units of $300 \mathrm{yr}$, and $I_{45}$ is its principal moment of inertia in units of $10^{45} \mathrm{~g} \mathrm{~cm}^{2}$. The indirect limit on $h_{0}$ is independent of frequency.

The choice of a fiducial age of $300 \mathrm{yr}$ for Cas A, at the young end of the range estimated by Fesen et al. (2006b), is conservative in that it gives a larger search parameter space (see Section 2.3). It also raises the indirect limit by $\sim 10 \%$, a small effect compared to the uncertainties in the distance (of order 10\%), and the principal moment of inertia, which may be up to three times higher than its fiducial value (see Abbott et al. 2007c). The uncertainties in the direct upper limits presented in Section 3 are on the order of $10 \%-15 \%$, due to uncertainties in the calibration of the LIGO detectors (of order $10 \%$; see Section 2.1), and systematic uncertainties in the search pipeline (of order 5\%; see Section 2.2). Equation (1) assumes gravitational waves from a mass quadrupole; the equivalent limit for $r$-modes is higher by tens of percent (Owen 2010).

The indirect limit on $h_{0}$ may be converted into an indirect limit on the equatorial ellipticity

$$
\epsilon \lesssim 3.9 \times 10^{-4} \tau_{300}^{-1 / 2} I_{45}^{-1 / 2} f_{100}^{-2},
$$

where $f_{100}$ is the gravitational-wave frequency in units of $100 \mathrm{~Hz}$. Due to the uncertainties in neutron star parameters mentioned above, this limit on $\epsilon$ is overall uncertain by roughly a factor two. The indirect limit on $h_{0}$ also implies an indirect limit on gravitational waves from $r$-modes (Owen 2010), in terms of their amplitude

$$
\alpha \lesssim 0.14 \tau_{300}^{-1 / 2} f_{100}^{-3},
$$

where the uncertainty due to the moment of inertia and other properties of the star, while more complicated, is roughly a factor of 2-3. If $\alpha$ varies over the observation time, this limit applies to the rms value of $\alpha$ over time. See Owen (2010) for precise definitions of $\alpha$ and $\epsilon$, translations to other quantities used in the literature (including $h_{0}$ ), and more discussion of uncertainties.

Wette et al. (2008) showed that a search for Cas A of 12 days of LIGO S5 data in the band $100-300 \mathrm{~Hz}$ is feasible and can expect to beat the indirect limits. The Cas A indirect limits on $h_{0}$ and $\epsilon$ are comparable to the spin-down limits for the Crab pulsar, which have been beaten by searches of LIGO S5 data (Abbott et al. 2008b, 2010). The indirect limits on $\alpha$ are lower and therefore more interesting than for the Crab if Cas A is emitting at $100-300 \mathrm{~Hz}$. The indirect limits are also comparable to the best upper limits achieved by all-sky searches for periodic gravitational waves (Abbott et al. 2009b, 2009c). The all-sky 
searches covered longer spin-down timescales and thus were not sensitive to Cas $\mathrm{A}$ if it is emitting gravitational waves near the indirect limit.

\section{SEARCH PIPELINE}

This section describes the gravitational-wave search for Cas A. The search pipeline consists of the selection of data for the search (Section 2.1), the analysis method (Section 2.2), the search parameter space (Section 2.3), the template bank used to compute the search (Section 2.4), post-processing of the results (Section 2.5), examination of the significance of the largest value of the detection statistic returned by the search (Section 2.6), and, in the event of no detection, calculation of the upper limits (Section 2.7).

\subsection{Data Selection from the LIGO S5 Run}

LIGO is a network of three interferometric detectors: a $4 \mathrm{~km}$ arm-length detector in Livingston, Louisiana (L1) and two detectors of $4 \mathrm{~km}(\mathrm{H} 1)$ and $2 \mathrm{~km}(\mathrm{H} 2)$ arm lengths co-located in Hanford, Washington.

The S5 science run (Abbott et al. 2009d) is LIGO's fifth and most recently completed science run. It commenced at 2005 November 4, 16:00 UTC at Hanford, and at 2005 November 14, 16:00 UTC at Livingston; it ended at 2007 October 1, 00:00 UTC. The S5 run collected over one year of science data coincident among all three detectors, with an overall triple-coincidence duty cycle of $54 \%$. Interruptions caused by environmental disturbances, as well as scheduled breaks for maintenance and commissioning of equipment, accounted for the downtime. During S5, the detectors were operating at very near their design sensitivities. The strain noise of the two $4 \mathrm{~km}$ detectors was on average less than $3 \times 10^{-23} \mathrm{~Hz}^{-1 / 2}$ at their most sensitive frequencies (around $140 \mathrm{~Hz}$ ) and less than $5 \times 10^{-23} \mathrm{~Hz}^{-1 / 2}$ over $100-300 \mathrm{~Hz}$, and generally improved (as did the duty cycle) over the course of the run.

This search uses science data from only the L1 and H1 detectors. Data from the $\mathrm{H} 2$ detector are less sensitive, but carry the same computational cost to search. A small percentage of the acquired science data are excluded by data quality controls, which identify times when the data are known to be unsuitable for analysis. This includes, e.g., data taken when the output photodiodes of an interferometer were saturated, when the calibration of the data was ill-defined, when high winds were measured at the Hanford observatory, and $30 \mathrm{~s}$ before an interferometer lost lock. The remaining science data are calibrated (Abbott et al. 2010, 2009d; Abadie et al. 2010) to produce a (discontinuous) time series of gravitational-wave strain, $h(t)$. The time series is then broken into 30 minute segments. Because not every continuous section of $h(t)$ is an integer multiple of 30 minutes in length, some science data are discarded in this process. Finally, each 30 minute segment is high-pass filtered above $40 \mathrm{~Hz}$ and Fourier transformed ${ }^{65}$ to form Short Fourier Transforms (SFTs) of $h(t)$. The SFTs are the input data to the search pipeline.

The maximum uncertainties in the calibration of $h(t)$ are $10.4 \%$ in amplitude and 4.5 in phase for $\mathrm{H} 1$, and $14.4 \%$ in amplitude and 4.2 in phase for L1; all uncertainties are constant in time to within 1\% (Abadie et al. 2010). The uncertainties in the calibration amplitude contribute to the overall systematic

\footnotetext{
65 Prior to the Fourier transform, each segment is multiplied by a nearly square Tukey window to mitigate transients at the start and end of the segment The loss in signal power due to the windowing is on the order of $0.1 \%$.
}

uncertainty in the upper limits presented in Section 3. The analysis method used in this search (see Section 2.2) is sensitive only to the relative phase uncertainty between detectors. Even for a worst-case relative phase uncertainty of $\sim 10^{\circ}$, the resulting difference in the signal frequency between detectors would still be much less than the mismatch allowed for by the search template bank (see Section 2.4). Thus, the phase uncertainties do not affect the results of this search.

The search is restricted to a data set spanning a maximum of 12 days (Wette et al. 2008). The computational cost of a coherent search for Cas A scales with the 7 th power of the timespan between the first and last timestamp of the analyzed SFTs, while the sensitivity scales only with the square root of the observation time. Increasing the timespan from 12 days would therefore rapidly increase the computational cost, for a negligible improvement in sensitivity. To select the 12 day data set, we compute a figure of merit for each possible data set spanning 12 days, chosen from the available S5 SFTs. The figure of merit, which is proportional to the estimated power signal-to-noise ratio, is given by $\sum_{k, f}\left[S_{h}(f)\right]^{-1}$, where $S_{h}$ is the strain noise power spectral density at frequency $f$ in the SFT numbered $k$, and the summation is over all SFTs in the data set and over the frequency band $100-300 \mathrm{~Hz}$. We select the data set with the maximum value of the figure of merit.

At the time this search was conducted, SFTs were available from the beginning of S5 until 2007 April 19 UTC. In this period, $\sim 9 \%$ of the $\mathrm{H} 1$ science data and $\sim 13 \%$ of the L1 science data are excluded, either due to data quality vetoes or due to the segmentation of $h(t)$ during SFT generation. The 12 day data set selected for the search begins at 2007 March 20, 20:56:37 UTC and ends at 2007 April 1, 20:50:04 UTC. It contains a total of 934 SFTs (445 from L1 and 489 from H1) and an average of 9.7 days of data from each detector.

\subsection{Analysis Method}

The data are searched using the $\mathcal{F}$-statistic, a coherent matched filtering technique used to search for periodic gravitational waves using multiple detectors (Jaranowski et al. 1998; Cutler \& Schutz 2005). Matched filtering requires an accurate model, or template, of the signal. The template models the response of a detector to the two polarizations ("+" and " $x$ ") of the gravitational-wave signal emitted by a rotating neutron star. In addition to the sky position, the barycentered gravitationalwave frequency, and its derivatives, the signal template has four parameters related to amplitude and polarization: the intrinsic strain $h_{0}$, initial phase constant $\phi_{0}$, inclination angle $\iota$ of the star's rotation axis to the line of sight, and polarization angle $\psi$. The $\mathcal{F}$-statistic is the logarithm of the likelihood ratio analytically maximized over these unknown parameters. The value of the $\mathcal{F}$-statistic is usually quoted as $2 \mathcal{F}$.

In transforming a signal from the reference frame of the star to that of the detector, the intrinsic frequency of the source is modulated by Doppler effects due to the sidereal and orbital motion of Earth with respect to the source. The transformation requires the right ascension $\alpha$ and declination $\delta$ of the star, which for Cas A are known to high precision: $\alpha=23^{\mathrm{h}} 23^{\mathrm{m}} 27^{\mathrm{s}} .943 \pm 0.05$, and $\delta=58^{\circ} 48^{\prime} 42^{\prime \prime} .51 \pm 0^{\prime \prime} .4$ (Fesen et al. 2006a). The sky resolution of the $\mathcal{F}$-statistic is, for a data set spanning 12 days, much coarser than the measured uncertainties in $\alpha$ and $\delta$ (Whitbeck 2006), and so no search over sky position is required. The search is conducted over the remaining unknown function in the signal template: the instantaneous frequency of the source as observed at the solar system barycenter, $f(t)$. This 
is modeled as

$$
f(t) \approx f+\dot{f}\left(t-t_{0}\right)+\frac{1}{2} \ddot{f}\left(t-t_{0}\right)^{2},
$$

where the initial frequency $f$, first spin-down $\dot{f}$, and second spin-down $\ddot{f}$ (all evaluated at the start time of the data set, $t=t_{0}$ ) constitute the search parameters. In previous searches for periodic gravitational waves, it has not been necessary to include a second spin-down, but one is required for this search due to the young age of Cas A (Wette et al. 2008).

The signal template does not allow for the possibility that Cas A glitched during the 12 days spanned by the data. On the other hand, even the most frequently glitching pulsar does so only a few times per year, and glitch population statistics do not clearly indicate that the youth of Cas A is sure to mean more frequent glitches (Yuan et al. 2010). The worst case would be a glitch at the midpoint of the 12 days span, since in other cases the template would pick up the longer of the pre- and post-glitch coherent stretches. Amplitude signal-to-noise accumulates as the square root of observation time, so the worst loss would be a factor of $\sim 2$.

The $\mathcal{F}$-statistic implicitly assumes a uniform prior on the polarization angle $\psi$, and therefore the standard data analysis and upper limit procedures remain valid for gravitational-waves signals from $r$-modes, even though the signal template used in this search assumes a mass quadrupole (Owen 2010).

The Cas A search uses the ComputeFStatistic_v2 implementation of the $\mathcal{F}$-statistic, which is available as part of the LALSuite software package. ${ }^{66}$ Values of the $\mathcal{F}$-statistic returned by ComputeFStatistic_v2 have an uncertainty of up to $5 \%$, due to practical computation issues and optimizations (see Prix 2010).

\subsection{Parameter Space}

The range of the gravitational-wave frequency, $100 \mathrm{~Hz} \leqslant$ $f \leqslant 300 \mathrm{~Hz}$, is chosen based on the estimate in Wette et al. (2008) of the frequency band over which a search of LIGO S5 data could beat the indirect limit on $h_{0}$ at reasonable computational cost.

The ranges of the spin-down parameters $\dot{f}$ and $\ddot{f}$ are chosen to be (Wette et al. 2008)

$$
-\frac{f}{\langle\min n-1\rangle \tau} \leqslant \dot{f} \leqslant-\frac{f}{\langle\max n-1\rangle \tau}
$$

and

$$
\frac{(\min n) \dot{f}^{2}}{f} \leqslant \ddot{f} \leqslant \frac{(\max n) \dot{f}^{2}}{f},
$$

respectively, where the braking index $n$ is defined below. The age of Cas A, $\tau$, is chosen to be $300 \mathrm{yr}$ (as discussed in Section 1.2). Note that the range of $\dot{f}$ depends on $f$, and the range of $\ddot{f}$ depends on both $f$ and $\dot{f}$. The resulting shape of the three-dimensional parameter space of $f, \dot{f}$, and $\ddot{f}$ is depicted in Figure 1.

The choices of $\dot{f}$ and $\ddot{f}$ ranges are motivated by the desire to cover a wide range of astrophysically motivated possibilities, expressed in terms of the braking index. In Equation (6), we use the definition of the instantaneous braking index $n=f \ddot{f} \dot{f}^{-2}$; in

\footnotetext{
66 The version of the software used in the search is tagged with the identifier S5CasASearch. See https://www.lsc-group.phys.uwm.edu/daswg/ projects/lalsuite.html
}

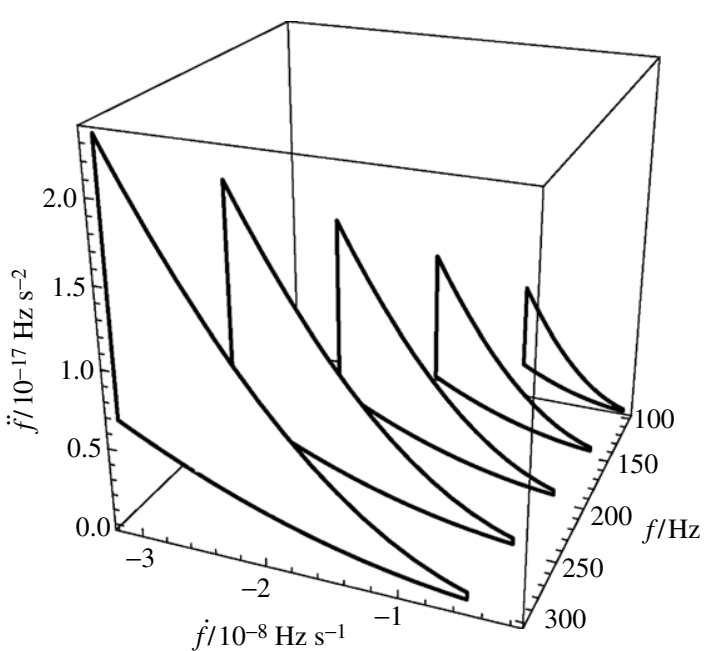

Figure 1. Visualization of the Cas A search parameter space. The black-outlined shapes are slices of the $\dot{f}-\ddot{f}$ parameter space at the following fixed values of $f$ : (back to front) 100, 150, 200, 250, and $300 \mathrm{~Hz}$.

Equation (5), the angled brackets denote the average value of $n$ over the lifetime of Cas A. If the dominant emission mechanism driving the spin-down of Cas A has changed over its lifetime, these two quantities will be different. Therefore, in the spirit of trying to cover the broadest imaginable parameter space, we do not constrain the instantaneous and averaged values of $n$ to be the same. Instead, we search over values of $n$ (both instantaneous and averaged) between 2 and 7. This range covers the possibilities that Cas $\mathrm{A}$ is spinning down primarily due to magnetic dipole radiation $(n=3)$ or to gravitational waves generated by a mass quadrupole $(n=5)$ or by constant- $\alpha r$ modes $(n=7)$. This range also covers the braking indices of nearly all known pulsars, which are typically between 2 and 3 (Livingstone et al. 2006). The exception is the Vela pulsar, for which $n \approx 1.4$ (Lyne et al. 1996). Extending $n$ to lower values would dramatically increase the computational cost of the search.

\subsection{Computation}

The search consists of computing the $\mathcal{F}$-statistic over a finite bank of templates, whose parameters are given by points within the search parameter space. The search uses a template bank generation algorithm (Wette 2009) which locates the parameter space points at the vertices of a body-centered cubic lattice. This minimizes the number of points per unit volume required to cover the parameter space (see, e.g., Conway \& Sloane 1988; Prix 2007b). The points are spaced using the $\mathcal{F}$-statistic parameter space metric (Whitbeck 2006; Prix 2007a) to ensure that the maximum expected fractional loss in signal-to-noise ratio (known as the mismatch) will never exceed 20\%. Due to strong correlations between the frequency and spin-down parameters and the irregular shape of the parameter space, it was necessary to place additional templates outside of the parameter space to fully cover the parameter space boundaries, particularly for $\ddot{f}$. As a result, the number of templates searched, $N \approx 7 \times 10^{12}$, is an order of magnitude larger than that estimated in Wette et al. (2008). This resulted in a greater computational cost but does not greatly affect the sensitivity of the search, which is only weakly dependent on the number of templates (see Section 2.6).

The search is divided into $\sim 21,500$ independent computational jobs by partitioning the range of $f$ into small bands, each 
Table 1

Frequency Bands Containing Spuriously Large Values of the $\mathcal{F}$

\begin{tabular}{lcc}
\hline \hline Frequency Band & $2 \mathcal{F}_{\max }$ & Origin \\
\hline $108.860 \pm 0.018$ & 90 & Pulsar hardware injection no. 3 \\
$119.877 \pm 0.019$ & 72 & Sideband of 60 Hz harmonic \\
$128.000 \pm 0.017$ & 56 & $16 \mathrm{~Hz}$ harmonic \\
$139.225 \pm 0.058$ & 70 & L1-only line \\
$139.510 \pm 0.017$ & 72 & L1-only line \\
$144.751 \pm 0.056$ & 110 & L1-only line \\
$179.812 \pm 0.018$ & 51 & Sideband of 60 Hz harmonic \\
$185.630 \pm 0.055$ & 59 & L1-only line \\
$193.005 \pm 0.046$ & 66 & Pulsar hardware injection no. 8 \\
$193.391 \pm 0.018$ & 73 & L1-only line \\
$209.265 \pm 0.017$ & 54 &
\end{tabular}

Notes. Frequency bands (Column 1) identified during post-processing as containing spuriously large values of the $\mathcal{F}$-statistic (the maximum of which are given in Column 2), and a brief description of their origin (Column 3 ). See the text for details.

with approximately equal numbers of templates, resulting in band widths of $1.8 \mathrm{mHz}$ (at $f=100 \mathrm{~Hz}$ ) to $20 \mathrm{mHz}$ (at $f=$ $300 \mathrm{~Hz}$ ). The search completed in $\sim 3.5$ days on $\sim 5000$ cores of the ATLAS computer cluster at the Max Planck Institute for Gravitational Physics (Albert Einstein Institute) in Hanover, Germany. From each search job, only the $0.01 \%$ of templates with the largest values of $2 \mathcal{F}$ were recorded.

\subsection{Post-processing}

Data from the LIGO detectors are known to contain stationary or nearly stationary spectral lines originating from instrumental and environmental noise. The $\mathcal{F}$-statistic depends upon a robust estimator of the power spectral density of the noise. The implementation used in this search (see Section 2.2) uses a spectral running median with a window size of 50 SFT bins, or $27.8 \mathrm{mHz}$ (Abbott et al. 2007a). Lines that are narrower than the median window size will remain in the data and may result in spuriously large values of the $\mathcal{F}$-statistic.

To identify frequency bands where the $\mathcal{F}$-statistic may have been contaminated in this manner, we search for prominent narrow lines in power spectra of the searched $\mathrm{H} 1$ and L1 data. This procedure identified 11 frequency bands, which are listed in Table 1, along with the largest value of the $\mathcal{F}$-statistic found in each band. Templates whose instantaneous frequency $f(t)$ at any time falls within any of these bands are excluded from the remainder of the search pipeline. Approximately $2 \times 10^{6}$ templates (a fraction $\sim 3 \times 10^{-6}$ of the total number of templates) are excluded in this manner. Four of the bands contain a largest value of $2 \mathcal{F}$ in the range 51-59, which would not be regarded as statistically significant gravitational-wave candidates (see Section 2.6).

At certain times during S5, 10 simulated periodic gravitational-wave signals were injected into the LIGO detectors at the hardware level by mechanically oscillating the detector mirrors (Abbott et al. 2008a). Four of the injections had frequencies within the search band. The Cas A search data set was not selected with regard to times when the hardware injections were active. As a result, $\lesssim 1$ day of the searched data $(\sim 4.2 \%$ of the $\mathrm{H} 1$ data, and $\sim 9.2 \%$ of the L1 data) contain the injections, and the effective intrinsic strains of the injections are reduced by a factor of $\sim 10$. After accounting for this reduction, two of the injections have strains of $h_{0} \lesssim 10^{-25}$ and are undetectable by this search. The remaining two injections (designated nos. 3 and 8 ) have reduced strains of $h_{0} \sim 1.63 \times 10^{-24}$ and $1.59 \times 10^{-24}$,

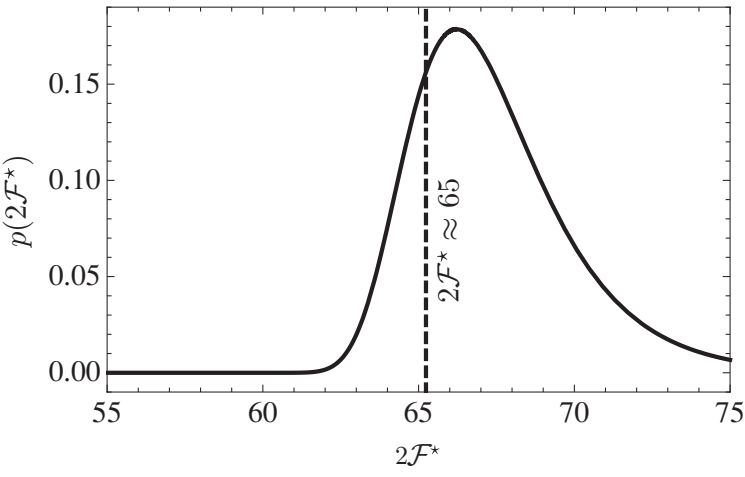

Figure 2. The solid line is the probability density of the largest value of the $\mathcal{F}$-statistic, $2 \mathcal{F}^{\star}$, under the assumption that no gravitational-wave signal from Cas A is present in the searched data (see Equation (7)). The dashed line denotes the value of $2 \mathcal{F}^{\star}$ returned by the Cas A search.

respectively. These injections are found by the Cas A search at frequencies consistent with the parameters of the injected signals (Wette 2009). Neither injection is at the sky position of Cas A, but are nevertheless detected due to their strength, and the poor sky localization of short-duration (i.e., less than 1 day) signals arising from global correlations in the signal parameter space (Prix \& Itoh 2005; Pletsch 2008)

The remaining nine non-injection bands are ruled out as gravitational-wave candidates for the following reasons. Two contain harmonics of the $60 \mathrm{~Hz}$ power mains frequency, and one contains a harmonic of the $16 \mathrm{~Hz}$ data acquisition buffering frequency (Abbott et al. 2004a). The remaining six bands contain narrow instrumental lines which occur only in the L1 detector. We would expect a convincing gravitational-wave candidate to be seen in both detectors. Four of these six lines (at $\sim 139.2 \mathrm{~Hz}, 144.7 \mathrm{~Hz}, 185.6 \mathrm{~Hz}$, and $193.0 \mathrm{~Hz}$ ) are definitively identified with environmental noise, by correlating the gravitational wave data with data from environmental monitors (e.g., magnetometers, accelerometers, and microphones). The sources of the remaining two lines, at $\sim 139.5 \mathrm{~Hz}$ and at $\sim 209.2 \mathrm{~Hz}$, are not conclusively identified. The value of the $\mathcal{F}$-statistic associated with the $\sim 209.2 \mathrm{~Hz}$ line, $2 \mathcal{F}=54$, is smaller than the largest value of $2 \mathcal{F}$ expected from the search in the absence of a gravitational-wave signal (see the next section) and is therefore not a candidate for a gravitational-wave signal from Cas A. The value of the $\mathcal{F}$-statistic associated with the $\sim 139.5 \mathrm{~Hz}$ line, $2 \mathcal{F}=72$, is also not statistically significant; as may be deduced from Figure 2, there is a $\sim 5 \%$ probability that the search would return a higher largest value of $2 \mathcal{F}$ without a gravitational-wave signal being present in the data.

\subsection{Significance of the Largest $2 \mathcal{F}$}

The largest value of the $\mathcal{F}$-statistic returned by the search after post-processing is denoted $2 \mathcal{F}^{\star}$. This is our most promising candidate for a gravitational-wave signal from Cas A. The probability density of $2 \mathcal{F}^{\star}$, under the assumption that no gravitational-wave signal from Cas A is present in the searched data, is given by

$$
p\left(2 \mathcal{F}^{\star}\right)=N p\left(\chi_{4}^{2} ; 2 \mathcal{F}^{\star}\right)\left[\int_{0}^{2 \mathcal{F}^{\star}} d(2 \mathcal{F}) p\left(\chi_{4}^{2} ; 2 \mathcal{F}\right)\right]^{N-1},
$$

where $N$ is the number of searched templates and $p\left(\chi_{4}^{2} ; 2 \mathcal{F}\right)$ denotes the probability density of a central $\chi^{2}$ distribution with 


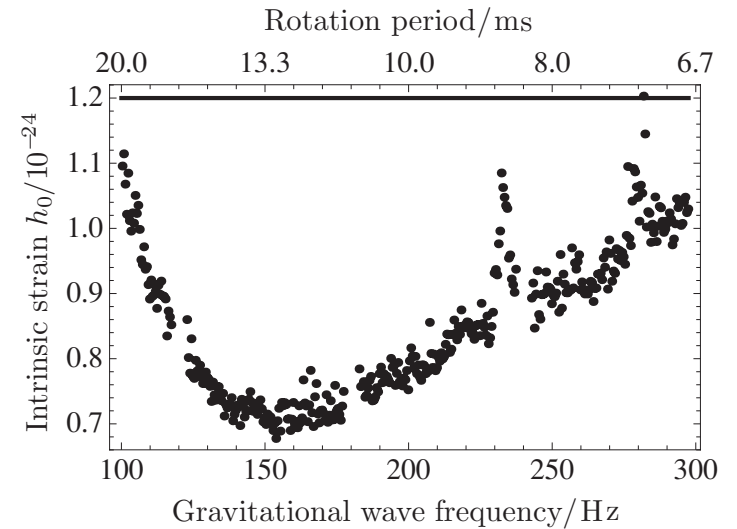

Figure 3. Upper limits at 95\% confidence (dots) on the intrinsic strain $h_{0}$ of gravitational waves from Cas A and the indirect limit (line). The gravitationalwave frequency is assumed to be twice the rotation frequency. Systematic uncertainties are not included; see Section 3 for discussion.

four degrees of freedom (i.e., the distribution of $2 \mathcal{F}$ in the absence of any signal and assuming Gaussian noise). If the value of $2 \mathcal{F}^{\star}$ returned by the Cas A search (see Section 3) is within the range of probable values expected from $p\left(2 \mathcal{F}^{\star}\right)$, it is not statistically significant. If, on the other hand, the value of $2 \mathcal{F}^{\star}$ is extremely unlikely to be drawn from $p\left(2 \mathcal{F}^{\star}\right)$, the candidate signal is worthy of further investigation.

Equation (7) assumes that each of the $N$ values of $2 \mathcal{F}$ is statistically independent. This is not strictly true, however, as we expect the $2 \mathcal{F}$ values of neighboring templates to be correlated due to the close template spacing. Therefore, in Equation (7), $N$ should be substituted with the number of statistically independent templates, $N_{i}<N$. An empirical estimate of the number of statistically independent templates found that $N_{i} \approx 0.88 N$ for this search (Wette 2009). A reduction in $N$ shifts the distribution of $p\left(2 \mathcal{F}^{\star}\right)$ toward lower values of $2 \mathcal{F}$, and thus increases the statistical significance of a candidate signal. For a reduction of $N$ to $0.88 N$, however, the shift is negligible, and can be ignored; indeed, Figure 2 plots Equation (7) with $N_{i}=N$. If, taking an extreme example, $N_{i}=0.1 N$, the position of the maximum of $p\left(2 \mathcal{F}^{\star}\right)$ would be shifted from $2 \mathcal{F} \sim 66$ to $\sim 62$ (see Figure 2), but the significance of the $2 \mathcal{F}^{\star}$ found by this search (see Section 3 ) would not be greatly increased. Therefore, the uncertainty in the number of statistically independent templates does not alter the significance of $2 \mathcal{F}^{\star}$, nor the conclusions reached in Section 3. The determination the number of statistically independent templates from first principles is an interesting area for further investigation.

\subsection{Upper Limits}

If, after examining $2 \mathcal{F}^{\star}$ (see Section 2.6), we conclude that no gravitational signal has been detected, we proceed to set $95 \%$ confidence upper limits on the intrinsic strain $h_{0}$, the ellipticity $\epsilon$, and the $r$-mode amplitude $\alpha$. The upper limits are determined using Monte Carlo injections, following the procedure described in Abbott et al. (2007a). The search frequency band is first partitioned into 400 sub-bands of width $0.5 \mathrm{~Hz}$; an upper limit is set separately for each sub-band. The choice of $0.5 \mathrm{~Hz}$ is small enough that the time-averaged noise floor of the detectors is approximately constant over each band and is large enough to keep the computational cost reasonable. We denote the largest values of $2 \mathcal{F}$ found by the full Cas A search in each sub-band by $2 \mathcal{F}_{\mathrm{s}-\mathrm{b}}^{\star}$; they serve as the false alarm thresholds

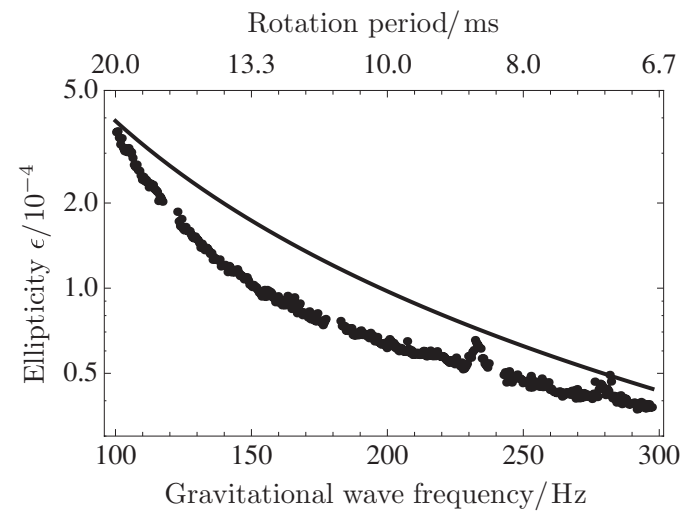

Figure 4. Upper limits at $95 \%$ confidence (dots) on the equatorial ellipticity $\epsilon$ of Cas A and the indirect limit (line). The gravitational-wave frequency is assumed to be twice the rotation frequency. Systematic uncertainties are not included; see Section 3 for discussion.

in each band. For each sub-band, a population of 5000-6000 periodic gravitational-wave signals, with $h_{0}$ fixed and all other parameters randomly chosen, are each in turn injected into the search data set and then searched for using the same analysis method used in the full Cas A search. We record the largest value of $2 \mathcal{F}$ found by the searches for each of the injected signals. The fraction of these $2 \mathcal{F}$ values that are greater than $2 \mathcal{F}_{\mathrm{s}-\mathrm{b}}^{\star}$ gives the confidence corresponding to the fixed value of $h_{0}$.

To expedite the injection procedure, we use an analytic model of the distribution of the population of injected signals to provide an educated guess at the value of $h_{0}$ required for $95 \%$ confidence (Wette 2009). We then perform the injection procedure once, at that $h_{0}$, to check that the recovered fraction really is $95 \%$. We find that the analytic model slightly overestimates the required $h_{0}$, and so the $95 \%$ confidence upper limits presented in Section 3 are conservative.

\section{RESULTS}

After post-processing, including the exclusion of the 11 frequency bands discussed in Section 2.5, the largest remaining value of the $\mathcal{F}$-statistic returned by the search is $2 \mathcal{F}^{\star} \approx 65$. Figure 2 compares $2 \mathcal{F}^{\star}$ to its expected theoretical distribution, under the assumption that the searched data contain no gravitational-wave signal from Cas A (see Section 2.6). It is clear that $2 \mathcal{F}^{\star}$ is consistent with this distribution and is therefore not statistically significant. We therefore conclude that the searched data do not contain any plausible gravitational-wave signal from Cas A. This conclusion is not significantly influenced by the uncertainty in the number of statistically independent templates (see Section 2.6).

Upper limits at $95 \%$ statistical confidence on $h_{0}, \epsilon$, and $\alpha$ are plotted, alongside their respective indirect limits, in Figures 3-5, respectively. Systematic uncertainties in the direct upper limits on $h_{0}$ are of order 10\%-15\% (see Sections 2.1 and 2.2). Systematic uncertainties in all three indirect limits, and in the direct upper limits on $\epsilon$ and $\alpha$ inferred from $h_{0}$, are roughly a factor 2-3 for $\alpha$ and 2 for the others (see Section 1.2). As expected, the upper limits beat the indirect limits over the gravitational-wave frequencies $100-300 \mathrm{~Hz}$. The equivalent ranges of rotation periods are 6.7-20 ms for the upper limits on $h_{0}$ and $\epsilon$ (which assume the gravitational-wave frequency to be twice the rotation frequency), and $4.4-13 \mathrm{~ms}$ for the upper limits on $\alpha$ (which assume a 4/3 ratio of gravitational-wave frequency to rotation frequency). Upper limits within $2 \mathrm{~Hz}$ of harmonics 


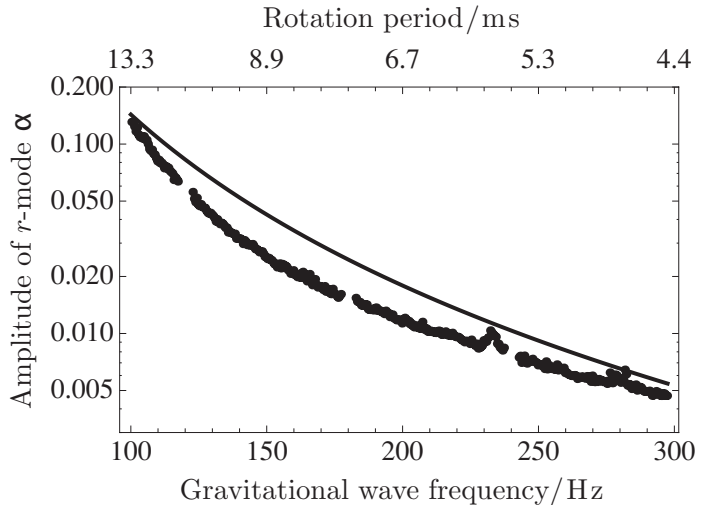

Figure 5. Upper limits at 95\% confidence (dots) on the amplitude $\alpha$ of $r$ mode oscillations of Cas A and the indirect limit (line). The gravitationalwave frequency is assumed to be $4 / 3$ times the rotation frequency. Systematic uncertainties are not included; see Section 3 for discussion.

of the $60 \mathrm{~Hz}$ power mains frequency are severely degraded due to noise and are excluded from the figures. Between 230 and $240 \mathrm{~Hz}$, and around $280 \mathrm{~Hz}$, the upper limits are degraded by disturbances in the broadband noise of the $\mathrm{H} 1$ detector.

\section{DISCUSSION}

The tightest upper limit on $h_{0}$ (Figure 3 ) is $\sim 7 \times 10^{-25}$ at $\sim 150 \mathrm{~Hz}$ in the region where the LIGO detectors are at their most sensitive. The search improved slightly upon the expected upper limits on $h_{0}$ estimated by Wette et al. (2008), due to slightly better detector sensitivity and duty cycle in the selected data set. Therefore, the search could have beaten the indirect limits at frequencies slightly outside of the $100-300 \mathrm{~Hz}$ band, although the computational cost increases rapidly at higher frequencies, and the noise floor of the detectors rises steeply at lower frequencies.

The upper limits on $\epsilon$ (Figure 4) range from $\sim 4 \times 10^{-4}$ at $100 \mathrm{~Hz}$ (20 ms rotation period) to $\sim 4 \times 10^{-5}$ at $300 \mathrm{~Hz}$ (6.7 ms), assuming the canonical parameters of Equation (2). The upper limits are higher than the maximum $\epsilon$ of a few times $10^{-6}$ predicted for normal neutron stars, even with recent results indicating a high breaking strain of the crust (Horowitz \& Kadau 2009). Ellipticities of a few times $10^{-4}$ are within the range of predictions (Owen 2005; Lin 2007; Haskell et al. 2007; Knippel \& Sedrakian 2009) for various forms of crystalline quark matter (Xu 2003; Mannarelli et al. 2007). Robust hybrid models (Glendenning 1992) could sustain ellipticities up to about $1 \times 10^{-4}$ (scaled from Owen 2005) if the breaking strain of Horowitz \& Kadau (2009) is valid for the mixed phase of matter. Ellipticities comparable to our upper limits could also be sustained by internal magnetic fields of order $10^{16} \mathrm{G}$, depending on the field configuration, equation of state, and superconductivity of the star (Cutler 2002; Haskell et al. 2008; Akgün \& Wasserman 2008; Colaiuda et al. 2008).

It is important to realize that upper limits on $\epsilon$ cannot be used to constrain properties of QCD or the composition of the neutron star, which may simply have an ellipticity much lower than the theoretical maximum. The upper limits on $\epsilon$ do, however, constrain the internal magnetic field to be less than of order $10^{16} \mathrm{G}$, if Cas A is spinning fast enough to radiate gravitational waves in the searched frequency band.

The upper limits on $\alpha$ (Figure 5) range from $\sim 0.14$ at $100 \mathrm{~Hz}$ (13 ms rotation period) to $\sim 0.005$ at $300 \mathrm{~Hz}(4.4 \mathrm{~ms})$, assuming the canonical parameters of Equation (3). If the $r$ - mode amplitude varies with time, our limits on $\alpha$ are rms values over the observing time and the indirect limits are rms values over the lifetime of the star. Our upper limits on $\alpha$ are within the range of runaway low-viscosity scenarios at all frequencies, and on the high end of the frequency band they are comparable to the finite-viscosity parametric instability thresholds which tend to serve as attractors for the evolution (Bondarescu et al. 2009).

In several years the advanced LIGO and Virgo interferometers are expected to be in operation, with sensitivities an order of magnitude better than data searched here and extending to lower frequencies. Extrapolating from these results, a similar search on data from advanced interferometers would be expected to be sensitive to ellipticities of a few times $10^{-6}$, which are achievable by neutron stars without exotic matter or by stars with internal magnetic fields less than $\sim 10^{14} \mathrm{G}$, or $r$-mode amplitudes a few times $10^{-4}$. More sophisticated data analysis methods, such as hierarchical methods, would further increase the sensitivity. There are also more young non-pulsing neutron stars and other astrophysically interesting objects that could be targeted by a search of this type.

The authors gratefully acknowledge the support of the United States National Science Foundation for the construction and operation of the LIGO Laboratory and the Science and Technology Facilities Council of the United Kingdom, the Max-PlanckSociety, and the State of Niedersachsen/Germany for support of the construction and operation of the GEO600 detector. The authors also gratefully acknowledge the support of the research by these agencies and by the Australian Research Council, the Council of Scientific and Industrial Research of India, the Istituto Nazionale di Fisica Nucleare of Italy, the Spanish Ministerio de Educación y Ciencia, the Conselleria d'Economia, Hisenda i Innovació of the Govern de les Illes Balears, the Royal Society, the Scottish Funding Council, the Scottish Universities Physics Alliance, The National Aeronautics and Space Administration, the Carnegie Trust, the Leverhulme Trust, the David and Lucile Packard Foundation, the Research Corporation, and the Alfred P. Sloan Foundation. This paper has been designated LIGO Document No. LIGO-P1000028-v7.

\section{REFERENCES}

Abadie, J., et al. 2010, Nucl. Inst. Meth. Phys. Res. A, in press (arXiv:1007.3973v1)

Abbott, B., et al. 2004a, Phys. Rev. D, 69, 122004

Abbott, B., et al. 2004b, Phys. Rev. D, 69, 082004

Abbott, B., et al. 2005a, Phys. Rev. D, 72, 102004

Abbott, B., et al. 2005b, Phys. Rev. Lett., 94, 181103

Abbott, B., et al. 2007a, Phys. Rev. D, 76, 082001

Abbott, B., et al. 2007b, Phys. Rev. D, 76, 082003

Abbott, B., et al. 2007c, Phys. Rev. D, 76, 042001

Abbott, B., et al. 2008a, Phys. Rev. D, 77, 022001

Abbott, B., et al. 2008b, ApJ, 683, L45 (erratum, 706, L203 [2009b])

Abbott, B., et al. 2009a, Phys. Rev. D, 79, 022001

Abbott, B. P., et al. 2009b, Phys. Rev. Lett., 102, 111102

Abbott, B. P., et al. 2009c, Phys. Rev. D, 80, 042003

Abbott, B. P, et al. 2009d, Rep. Prog. Phys., 72, 076901

Abbott, B. P., et al. 2010, ApJ, 713, 671

Akgün, T., \& Wasserman, I. 2008, MNRAS, 383, 1551

Arras, P., Flanagan, E. E., Morsink, S. M., Schenk, A. K., Teukolsky, S. A., \& Wasserman, I. 2003, ApJ, 591, 1129

Bondarescu, R., Teukolsky, S. A., \& Wasserman, I. 2009, Phys. Rev. D, 79 104003

Chakrabarty, D., Pivovaroff, M. J., Hernquist, L. E., Heyl, J. S., \& Narayan, R. 2001, ApJ, 548, 800

Colaiuda, A., Ferrari, V., Gualtieri, L., \& Pons, J. A. 2008, MNRAS, 385, 2080

Conway, J. H., \& Sloane, N. J. A. 1988, Sphere Packings, Lattices and Groups (New York: Springer) 
Cutler, C. 2002, Phys. Rev. D, 66, 084025

Cutler, C., \& Schutz, B. F. 2005, Phys. Rev. D, 72, 063006

De Luca, A. 2008, in AIP Conf. Proc. 983, 40 Years of Pulsars: Millisecond Pulsars, Magnetars and More, ed. C. Bassa et al. (Melville, NY: AIP), 311

Dwek, E., \& Arendt, R. G. 2008, ApJ, 685, 976

Fesen, R. A., Pavlov, G. G., \& Sanwal, D. 2006a, ApJ, 636, 848

Fesen, R. A., et al. 2006b, ApJ, 645, 283

Glendenning, N. K. 1992, Phys. Rev. D, 46, 1274

Gotthelf, E. V., \& Halpern, J. P. 2008, in AIP Conf. Proc. 983, 40 Years of Pulsars: Millisecond Pulsars, Magnetars and More, ed. C. Bassa et al. (Melville, NY: AIP), 320

Gotthelf, E. V., \& Halpern, J. P. 2009, ApJ, 695, L35

Halpern, J. P., \& Gotthelf, E. V. 2010, ApJ, 709, 436

Haskell, B., Andersson, N., Jones, D. I., \& Samuelsson, L. 2007, Phys. Rev. Lett., 99, 231101

Haskell, B., Samuelsson, L., Glampedakis, K., \& Andersson, N. 2008, MNRAS, 385,531

Ho, W. C. G., \& Heinke, C. O. 2009, Nature, 462, 71

Horowitz, C. J., \& Kadau, K. 2009, Phys. Rev. Lett., 102, 191102

Hwang, U., et al. 2004, ApJ, 615, L117

Jaranowski, P., Królak, A., \& Schutz, B. F. 1998, Phys. Rev. D, 58, 063001

Kaplan, D. L., Kulkarni, S. R., \& Murray, S. S. 2001, ApJ, 558, 270

Kim, Y., Rieke, G. H., Krause, O., Misselt, K., Indebetouw, R., \& Johnson, K. E. 2008, ApJ, 678, 287

Knippel, B., \& Sedrakian, A. 2009, Phys. Rev. D, 79, 083007

Krause, O., Birkmann, S. M., Usuda, T., Hattori, T., Goto, M., Rieke, G. H., \& Misselt, K. A. 2008, Science, 320, 1195

Krause, O., et al. 2005, Science, 308, 1604

Lin, L.-M. 2007, Phys. Rev. D, 76, 081502

Lindblom, L., Owen, B. J., \& Ushomirsky, G. 2000, Phys. Rev. D, 62, 084030

Livingstone, M. A., Kaspi, V. M., Gotthelf, E. V., \& Kuiper, L. 2006, ApJ, 647, 1286

Lyne, A. G., Pritchard, R. S., Graham-Smith, F., \& Camilo, F. 1996, Nature, 381,497
Mannarelli, M., Rajagopal, K., \& Sharma, R. 2007, Phys. Rev. D, 76, 074026 McLaughlin, M. A., Cordes, J. M., Deshpande, A. A., Gaensler, B. M., Hankins, T. H., Kaspi, V. M., \& Kern, J. S. 2001, ApJ, 547, L41

Mereghetti, S., Tiengo, A., \& Israel, G. L. 2002, ApJ, 569, 275

Murray, S. S., Ransom, S. M., Juda, M., Hwang, U., \& Holt, S. S. 2002, ApJ, 566,1039

Owen, B. J. 2005, Phys. Rev. Lett., 95, 211101

Owen, B. J. 2010, Phys. Rev. D, submitted (arXiv:1006.1994v1)

Owen, B. J., Lindblom, L., Cutler, C., Schutz, B. F., Vecchio, A., \& Andersson, N. 1998, Phys. Rev. D, 58, 084020

Pavlov, G. G., \& Luna, G. J. M. 2009, ApJ, 703, 910

Pavlov, G. G., Zavlin, V. E., Aschenbach, B., Trümper, J., \& Sanwal, D. 2000, ApJ, 531, L53

Pletsch, H. J. 2008, Phys. Rev. D, 78, 102005

Prix, R. 2007a, Phys. Rev. D, 75, 023004

Prix, R. 2007b, Class. Quantum Grav., 24, S481

Prix, R. 2010, The F-statistic and its Implementation in ComputeFStatistic v2, Tech. Rep. LIGO-T0900149-v2, LIGO, available at http://dcc.ligo.org

Prix, R., \& Itoh, Y. 2005, Class. Quantum Grav., 22, S1003

Reed, J. E., Hester, J. J., Fabian, A. C., \& Winkler, P. F. 1995, ApJ, 440, 706

Reynolds, S. P., Borkowski, K. J., Green, D. A., Hwang, U., Harrus, I., \& Petre, R. 2008, ApJ, 680, L41

Ryan, E., Wagner, R. M., \& Starrfield, S. G. 2001, ApJ, 548, 811

Tananbaum, H. 1999, IAU Circ., 7246, 1

Wang, Z., Kaplan, D. L., \& Chakrabarty, D. 2007, ApJ, 655, 261

Wette, K. 2009, PhD thesis, The Australian National Univ.

Wette, K., et al. 2008, Class. Quantum Grav., 25, 235011

Whitbeck, D. M. 2006, PhD thesis, The Pennsylvania State Univ.

Wu, Y., Matzner, C. D., \& Arras, P. 2001, ApJ, 549, 1011

Xu, R. X. 2003, ApJ, 596, L59

Young, P. A., et al. 2006, ApJ, 640, 891

Yuan, J. P., Wang, N., Manchester, R. N., \& Liu, Z. Y. 2010, MNRAS, 404, 289 